\title{
Metastatic Anal Canal Carcinoma
}

National Cancer Institute

\section{Source}

National Cancer Institute. Metastatic Anal Canal Carcinoma. NCI Thesaurus. Code C5612.

An anal canal carcinoma which has spread to another anatomic site. 